\title{
Rectal potential difference and histology in Crohn's disease
}

\author{
W. S. J. RUDDELL, L. M. BLENDIS, AND D. LOVELL \\ From the Departments of Gastroenterology and Histopathology, Central Middlesex Hospital, London
}

SUMmaRY The rectal potential difference (PD) was measured in 27 patients with Crohn's disease, and in 16 subjects without gastrointestinal disease to establish a normal range. Sigmoidoscopic assessment and rectal biopsy were performed in all patients with Crohn's disease, and the mean resting rectal PD was significantly reduced in patients with sigmoidoscopically active disease and in those with abnormalities of the superficial epithelium on rectal biopsy. Patients with diarrhoea had a significantly lower mean resting PD than those with normal bowel habit, suggesting that an abnormality of rectal sodium transport may be contributing to the diarrhoea in these patients. The response of rectal PD to mineralocorticoid stimulation with oral fludrocortisone was measured in 13 patients. The PD failed to rise only in patients with sigmoidoscopically active disease, and the test proved to be a less sensitive indication of minor mucosal abnormalities than sigmoidoscopy or biopsy.

The normal rectal mucosa is electrically polarised, with the mucosal, or luminal side, negative with respect to the serosa (Edmonds and Godfrey, 1970). This potential difference (PD) arises in the superficial epithelium, is unaffected by removal of muscle coat and serosa (Binder and Rawlins, 1973), and represents the algebraic sum of individual PDs contributed by osmotic gradients, diffusion potentials, and active transfer potentials (Edmonds, 1975). In vitro studies have shown that active transport of sodium is the principal source of the PD in animals (Cooperstein and Brockman, 1959) and man (Grady et al., 1970). As conservation of salt and water is the principal function of colon and rectum (Devroede and Phillips, 1969; Edmonds, 1971), the transmucosal PD can be regarded as an important index of colonic function, and, specifically, of epithelial function. A fall in rectal PD occurs in acute colitis (Edmonds and Pilcher, 1970), due to cellular injury and damage to the sodium pump, and also to increased epithelial permeability to sodium (Rask-Madsen, 1973).

Mineralocorticoid stimulation increases the rectal PD by promoting sodium absorption. A raised rectal PD is found in hyperaldosteronism and may be used as a screening test for this condition (Edmonds and Richards, 1970). Exogenously administered mineralocorticoids also cause a substantial increase in rectal PD (Edmonds and Godfrey,

Received for publication 10 December 1976
1970) and the measurement of rectal PD before and after fludrocortisone has been suggested as a test of epithelial function (Edmonds, 1975).

In this study we have measured the rectal PD in a group of patients with Crohn's disease, and related it to the sigmoidoscopic appearance of the rectum and the histological appearance of a rectal biopsy. We have also studied the effect on rectal PD of oral fludrocortisone in relation to sigmoidoscopic and biopsy appearances.

\section{Methods}

Twenty-seven patients with Crohn's disease were studied. Eleven had undergone bowel resections and the diagnosis was confirmed by histology. In the remaining 16 patients the diagnosis was established by clinical, radiological, endoscopic, and biopsy findings. Patients were considered to have a normal bowel habit if they had three or fewer bowel actions per day with a formed motion, and to have diarrhoea if they regularly passed four or more loose stools per day. An additional 16 subjects without gastrointestinal disease were studied to establish a normal range of rectal PD. These subjects were normal volunteers and patients in whom gastrointestinal disease was excluded by radiology, sigmoidoscopy and rectal biopsy.

MEASUREMENT OF PD

The rectal PD was measured at $10 \mathrm{~cm}$ from the 
anus using the method of Archampong and Edmonds (1972). Briefly, the PD is measured between a probe electrode placed directly on the rectal mucosa through the sigmoidoscope and a reference electrode placed on an intradermal bleb of saline. Measured in this way the PD is stable and reproducible, and the voltage is read from a portable battery powered millivoltmeter with high input impedance (Searle Ltd., High Wycombe). Raising the bleb of saline has been shown to abolish skin PD for up to 30 minutes, so that the PD corresponds accurately to the PD measurement obtained using an intravenous reference electrode, and is thus a true reflection of transmucosal PD (Archampong and Edmonds, 1972). The exploring and reference electrodes incorporate a silver/silver chloride junction with an electrolyte bridge of $150 \mathrm{mM}$ sodium chloride in $4 \%$ agar. The procedure is performed at routine sigmoidoscopy, takes about five minutes and causes no added discomfort to the patient. The probe electrode was always placed on the mucosa under direct vision. The PD measurements and rectal biopsies were taken from non-ulcerated areas as near to $10 \mathrm{~cm}$ from the anal verge as possible. At each examination six to eight separate readings were taken from the same area of rectal mucosa, and the transmucosal PD was taken as the mean of these results.

\section{SIGMOIDOSCOPIC ASSESSMENT}

A simple classification of the sigmoidoscopic appearances into normal and abnormal was made. The abnormal appearances were further divided into active and inactive. Activity was defined by the presence of fissures, ulcers, friability, or spontaneous bleeding. The inactive mucosa had a diminished or absent vascular pattern and/or a granular appearance, without features of activity.

\section{RECTAL BIOPSY}

All the patients with Crohn's disease had a rectal biopsy taken under direct vision from the same area of mucosa immediately after the PD measurement. The normal controls for rectal PD were not biopsied. The same histopathologist assessed all the biopsies without knowledge of clinical details or sigmoidoscopic appearances. An overall assessment of the biopsies as normal or abnormal was made. Those judged to be abnormal were classified according to whether the abnormality involved the superficial epithelium, glandular epithelium, or lamina propria. Particular attention was paid to the 10 histological features listed in the Table.

\section{RESPONSE TO FLUDROCORTISONE}

In 13 of the patients with Crohn's disease the rectal
Table Histological abnormalities sought in superficial epithelium, glands and lamina propria of rectal biopsies

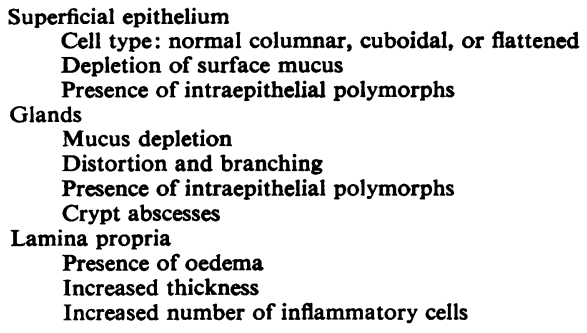

PD was measured before and after the oral administration of fludrocortisone $\mathbf{0 . 1} \mathrm{mg}$ eight hourly for six doses. The second PD measurement was made two to four hours after the last dose.

All studies were approved by the Hospital Ethics Committee, and informed consent was given by all subjects. Student's $t$ test for paired or unpaired data was used to assess the significance of differences between groups.

\section{Results}

The rectal PD in the 16 normal controls was $38 \pm 3 \mathrm{mV}$ (mean $\pm \mathrm{SE}$ ) mucosa negative to serosa. The normal range, as defined by mean \pm 2 SD was $28-48 \mathrm{mV}$.

\section{PD AND SIGMOIDOSCOPY}

Of the 27 patients with Crohn's disease 11 had a normal sigmoidoscopic appearance and the rectal $\mathrm{PD}$ in this group, $36 \pm 2 \mathrm{mV}$ (mean $\pm \mathrm{SE}$ ), was not significantly different from that in 10 patients with abnormal but inactive mucosa, $40 \pm 2 \mathrm{mV}$, or the normal controls. In contrast, the PD in six patients with sigmoidoscopically active rectal Crohn's disease, $17 \pm 3 \mathrm{mV}$, was significantly lower than both Crohn's patients with normal sigmoidoscopic appearances $(P<0.01)$ and those with an abnormal but inactive appearance $(P<0.01)$ (Fig. 1), and was also significantly lower than the normal controls $(P<0.01)$.

\section{PD AND BOWEL HABIT}

Excluding the six patients with sigmoidoscopically active rectal Crohn's disease, which we have shown to be associated with a low PD, the remaining 21 patients were subdivided according to bowel habit. The mean rectal PD in six Crohn's patients with diarrhoea, $29 \pm 3 \mathrm{mV}$, was significantly lower than that in 15 Crohn's patients with normal bowel habit, $41 \pm 2 \mathrm{mV}(\mathrm{P}<0.01)$ and than the normal 


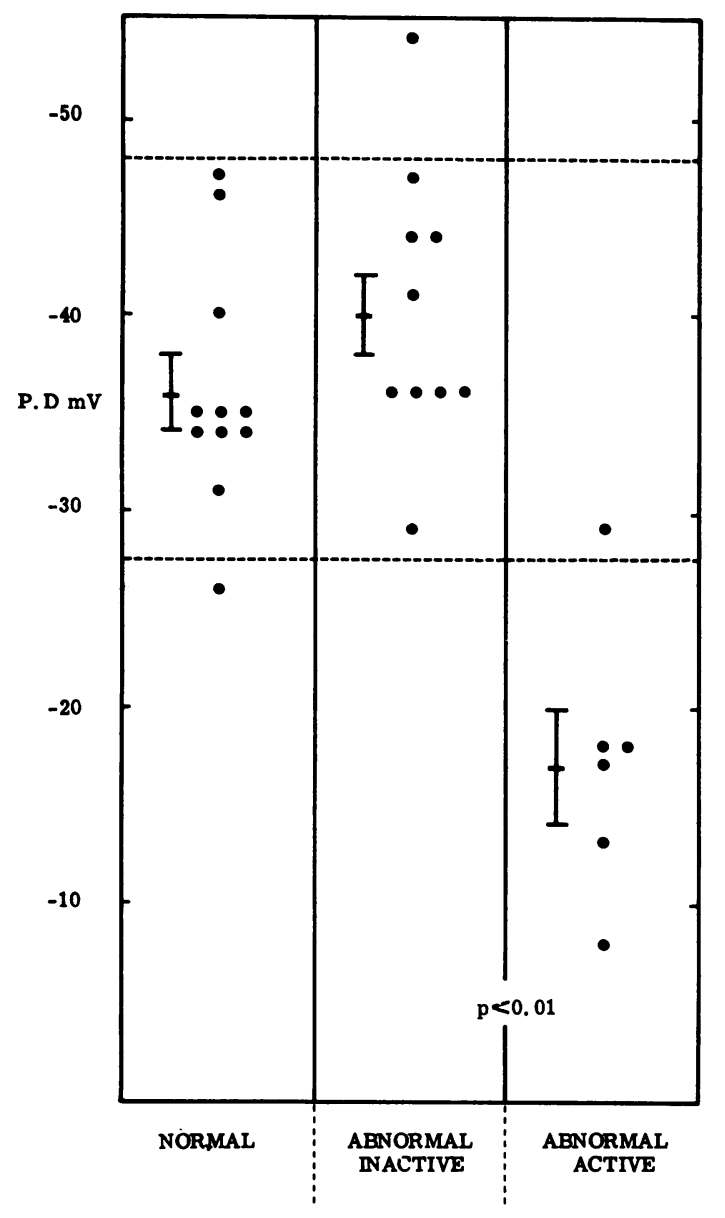

Fig. 1 Rectal $P D$ in $m V($ mean $\pm S E)$ in 27 patients with Crohn's disease, according to sigmoidoscopic appearance. Dotted lines represent the normal range of rectal $P D$.

controls $(P<0.01)$. Of the 21 patients without sigmoidoscopically active rectal disease, nine had undergone previous surgery involving resection of the terminal ileum, and the mean PD in this group, $36 \pm 2 \mathrm{mV}$, did not differ significantly from that in 12 patients who had not had resections, $39 \pm 2 \mathrm{mV}$.

\section{PD AND HISTOLOGY}

The PD results in the patients with Crohn's disease were related to the histological appearances of the rectal biopsies, irrespective of bowel habit. The mean PD in 10 patients with histologically normal rectal biopsies, $37 \pm 2 \mathrm{mV}$, was not significantly different from that in 11 patients in whom the histological abnormalities were confined to the lamina propria and glands, PD $39 \pm 2 \mathrm{mV}$. However, the rectal PD in six"patients whose biopsies showed abnormalities of the superficial epithelium, $18 \pm 4 \mathrm{mV}$, was significantly lower than both the group with normal histology $(P<0.01)$ and those in whom the histological abnormalities were restricted to the lamina propria and glands $(\mathrm{P}<0.01)$ (Fig. 2$)$.

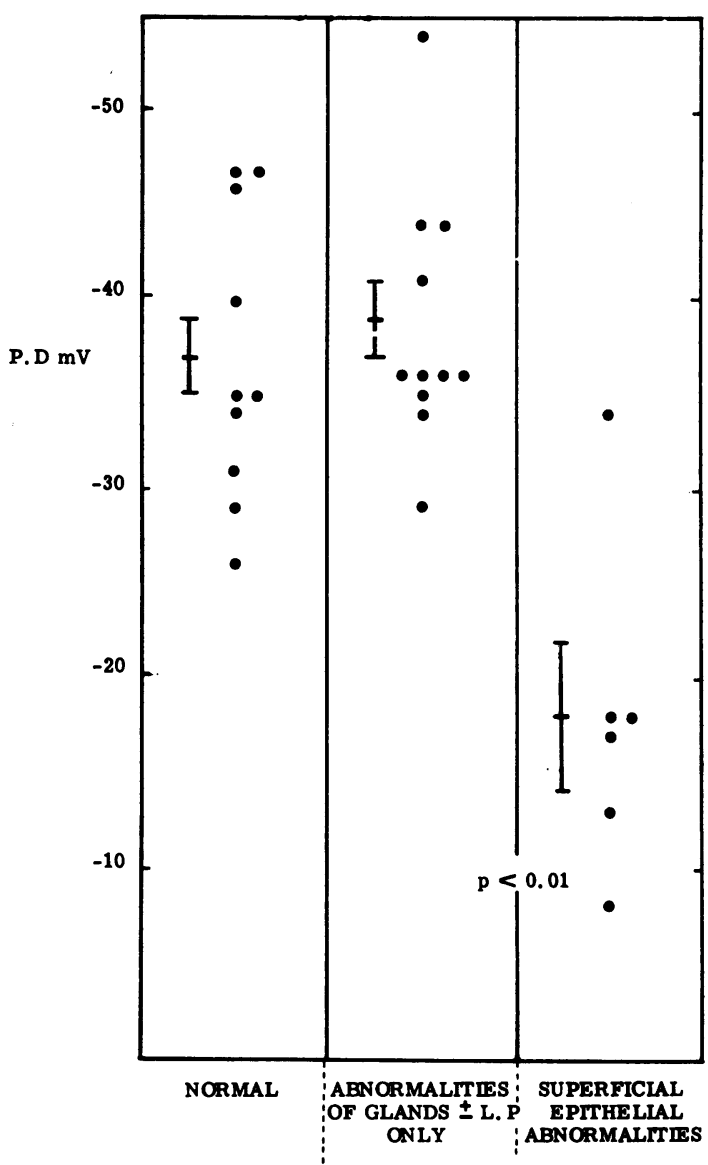

Fig. 2 Rectal $P D$ in $m V$ (mean $\pm S E$ ) in 27 patients with Crohn's disease, according to histological appearance of rectal biopsy.

\section{STUDIES WITH FLUDROCORTISONE}

As a test of epithelial function the response of the rectal PD to oral fludrocortisone was assessed in 13 of the 27 patients with Crohn's disease. In all six patients with normal sigmoidoscopic appearances the rectal PD rose after fludrocortisone by at least $12 \mathrm{mV}$ (mean rise $20 \pm 2 \mathrm{mV}$ ). In four of the seven patients with sigmoidoscopic abnormalities the PD rose by a similar amount (Fig. 3). Of these four patients two had sigmoidoscopic features of activity, and two were inactive. All three patients in whom the 


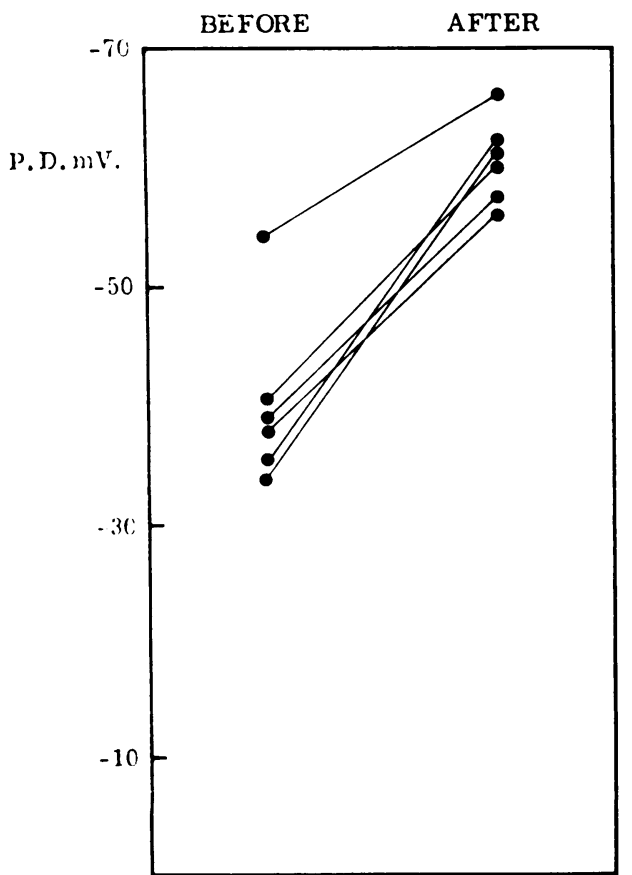

NORMAL SIGMOIDOSCOPY

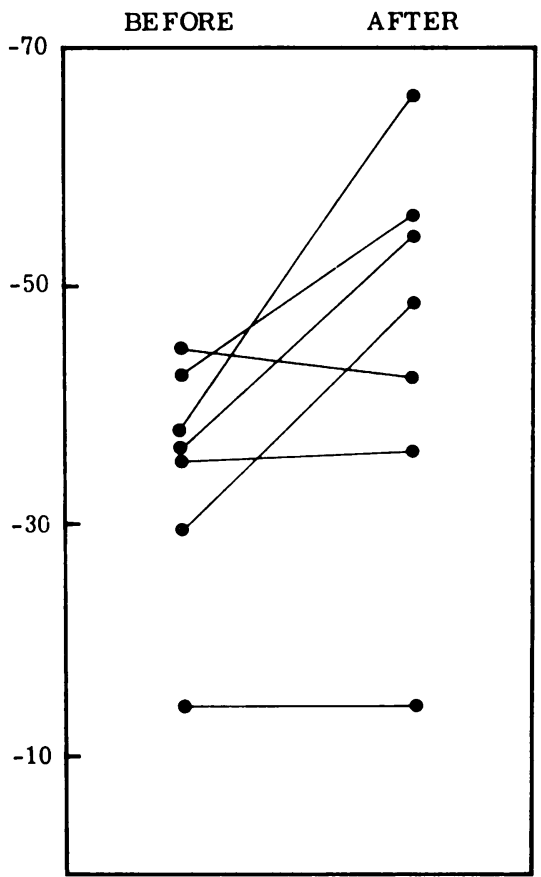

ABNORMAL SIGMOIDOSCOPY

Fig. 3 Rectal $P D$ in $m V$ before and after oral fludrocortisone in 13 patients with Crohn's disease with and without sigmoidoscopic abnormalities.

PD did not change after fludrocortisone had sigmoidoscopically active disease.

Only four of the 13 patients given fludrocortisone had a histologically normal rectal biopsy. In these four a significant rise $(P<0.01)$ of $P D$ was recorded, with a mean rise of $21 \pm 2 \mathrm{mV}$ (Fig. 4). The remaining nine patients had an abnormal biopsy, and of these six responded to fludrocortisone with a rise in PD, while in three there was no change (Fig. 4). Of the six responders two had histological abnormalities of the superficial epithelium, and in four the abnormality was confined to glands and/or lamina propria. All the three subjects with no rise in rectal PD after fludrocortisone had severe superficial epithelial abnormalities in their biopsies.

\section{Discussion}

A systematic study of rectal PD in Crohn's disease in relation to sigmoidoscopic and histological evidence of local disease has not previously been reported. The sigmoidoscopic criteria of activity commonly applied in ulcerative colitis (Baron et al., 1964) are inappropriate in Crohn's disease because of the patchiness of the abnormalities. For this reason we have classified the abnormal rectum into two categories only, active and inactive. Our normal range for rectal PD $(28-48 \mathrm{mV})$ is similar to that previously reported by Archampong and Edmonds (1972).

The unstimulated rectal PD was abnormally low in Crohn's disease only in the presence of severe sigmoidoscopic abnormalities, while an abnormal but 'inactive' mucosa was not associated with abnormal PD. Patients with low rectal PD all had abnormalities of the superficial epithelium on rectal biopsy. This observation supports in vitro studies of colonic mucosa which show that transmucosal PD is generated by the superficial epithelium during sodium transport (Binder and Rawlins, 1973). Histological abnormalities restricted to the glandular epithelium and lamina propria were not associated with a low unstimulated PD, suggesting that measurement of this variable in Crohn's disease is less sensitive than rectal biopsy for detecting minor mucosal abnormalities.

Patients with persistent diarrhoea but without sigmoidoscopically active disease had a significantly lower rectal PD than those with a normal bowel habit. More of the patients with diarrhoea were on 


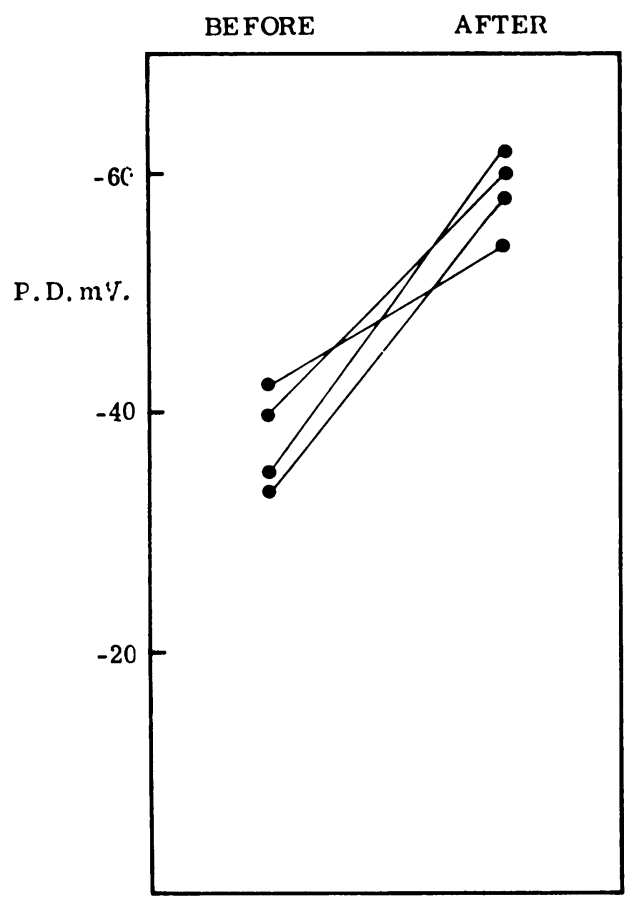

NORMAL HISTOLOGY

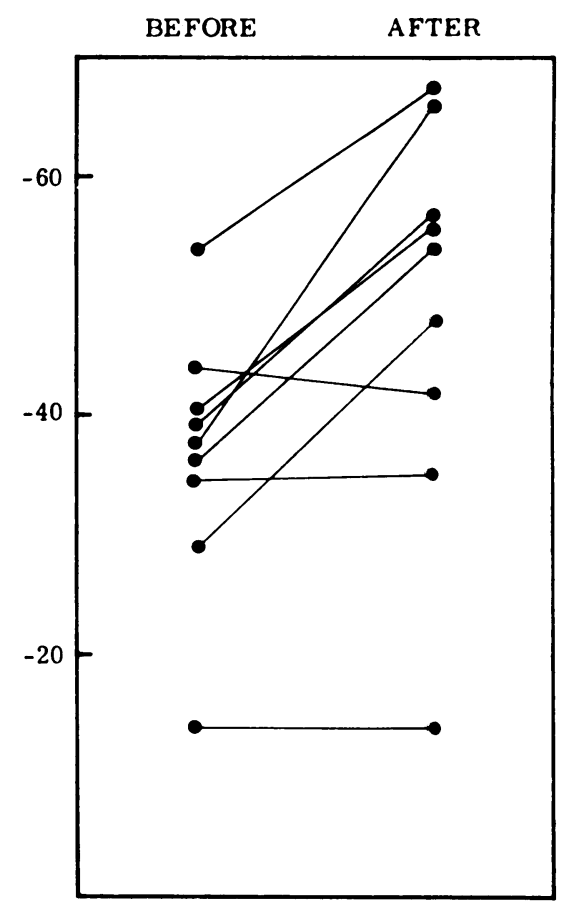

ABNORMAL HISTOLOGY

Fig. 4 Rectal $P D$ in $m V$ before and after oral fludrocortisone in 13 patients with Crohn's disease according to histological appearance of rectal biopsy.

steroid therapy than those with normal bowel habit but this would, if anything, tend to raise the PD. The lower PD found in patients with diarrhoea could be a result of altered sodium absorption produced by changes in colonic luminal environment. Extensive ileal disease or resection can produce malabsorption of bile salts (Austad et al., 1967) and fatty acids (Hofmann and Poley, 1972) resulting in an increased colonic load of dihydroxy bile acids and hydroxy fatty acids, both of which can produce inhibition of colonic sodium absorption (Mekhjian and Phillips, 1970; Bright-Asare and Binder, 1973). Altered colonic bile acid concentration is an unlikely explanation for the low PD, however, as there was no significant reduction in PD in patients who had undergone ileal resection. An alternative explanation is that we are measuring a primary abnormality of epithelial function, and that in these patients with diarrhoea there is an underlying impairment of rectal sodium transport, manifested as a reduced PD, which may be contributing to the diarrhoea by increasing faecal electrolyte losses (Giller and Phillips, 1972). The concept of a diffuse functional defect throughout the gastrointestinal tract in
Crohn's disease is supported by recent reports of widespread histological and biochemical abnormalities (Ferguson et al., 1975; Goodman et al., 1976) and the finding of reduced bidirectional sodium flux in apparently uninvolved bowel (Allan et al., 1975).

The normal response of the rectal mucosal PD to oral fludrocortisone is an increase of about $20 \mathrm{mV}$ as a result of mineralocorticoid stimulation of rectal sodium absorption (Edmonds, 1970). In ulcerative colitis a reduced or absent response to fludrocortisone may occur, even when the resting $\mathrm{PD}$ is within the normal range, presumably indicating persistent impairment of epithelial function (Edmonds, 1970). This suggests that minor epithelial abnormalities may be detected by a failure to respond to fludrocortisone even in the presence of a normal unstimulated rectal PD. Our results show that a normal response to fludrocortisone may occur despite both sigmoidoscopic and histological abnormalities, including abnormalities of the superficial epithelium. All patients in whom the histological abnormalities were confined to the glandular epithelium and lamina propria 
showed a normal PD rise. Thus the response of the rectal PD to oral fludrocortisone, employed as a dynamic test of epithelial function in Crohn's disease, is abnormal only in patients in whom rectal involvement is both macroscopically and microscopically severe and does not reliably distinguish patients in whom minor mucosal abnormalities are apparent by sigmoidoscopy and biopsy.

We would like to thank Dr T. D. Kellock and Dr J. J. Misiewicz for helpful advice and criticism and for allowing us to study their patients.

\section{References}

Allan, R., Steinberg, D. M., Dixon, K., and Cooke, W. T. (1975). Changes in the bidirectional sodium flux across the intestinal mucosa in Crohn's disease. Gut, 16, 201-204.

Archampong, E. Q., and Edmonds, C. J. (1972). Effect of luminal ions on the transepithelial electrical potential difference of human rectum. Gut, 13, 559-565.

Austad, W. I., Lack, L., and Tyor, M. P. (1967). Importance of bile acids and of an intact distal small intestine for fat absorption. Gastroenterology, 52, 638-646.

Baron, J. H., Connell, A. M., and Lennard-Jones, J. E. (1964). Variation between observers in describing mucosal appearances in proctocolitis. British Medical Journal, 1, 89-92.

Binder, H. J., and Rawlins, C. L. (1973). Effect of conjugated dihydroxy bile salts on electrolyte transport in rat colon. Journal of Clinical Investigation, 52, 1460-1466.

Bright-Asare, P., and Binder, H. J. (1973). Stimulation of colonic secretion of water and electrolytes by hydroxy fatty acids. Gastroenterology, 64, 81-88.

Cooperstein, I. L., and Brockman, S. K. (1959). The electrical potential difference generated by the large intestine: its relation to electrolyte and water transfer. Journal of Clinical Investigation, 38, 435-442.

Devroede, G. J., and Phillips, S. F. (1969). Conservation of sodium, chloride, and water by the human colon. Gastroenterology, 56, 101-109.
Edmonds, C. J. (1970). Electrical potential of the sigmoid colon and rectum in irritable bowel syndrome and ulcerative colitis. Gut, 11, 867-874.

Edmonds, C. J. (1971). Absorption of sodium and water by human rectum measured by a dialysis method. Gut, 12, 356-362.

Edmonds, C. J. (1975). Electrical potential difference of colonic mucosa. Gut, 16, 315-318.

Edmonds, C. J., and Godfrey, R. C. (1970). Measurement of electrical potential of the human rectum and pelvic colon in normal and aldosterone-treated patients. Gut, 11, 330337.

Edmonds, C. J., and Pilcher, D. (1973). Electrical potential difference and sodium and potassium fluxes across rectal mucosa in ulcerative colitis. Gut, 14, 784-789.

Edmonds, C. J., and Richards, P. (1970). Measurement of rectal electrical potential difference as an instant screening test for hyperaldosteronism. Lancet, 2, 624-627.

Ferguson, R., Allan, R. N., and Cooke, W. T. (1975). A study of the cellular infiltrate of the proximal jejunal mucosa in ulcerative colitis and Crohn's disease. Gut, 16, 205-208.

Giller, J., and Phillips, S. F. (1972). Electrolyte absorption and secretion in the human colon. American Journal of Digestive Diseases, 17, 1003-1011.

Goodman, M. J., Skinner, J. M., and Truelove, S. C. (1976). Abnormalities in the apparently normal bowel mucosa in Crohn's disease. Lancet, 1, 275-278.

Grady, G. F., Duhamel, R. C., and Moore, E. W. (1970). Active transport of sodium by human colon in vitro. Gastroenterology, 59, 583-588.

Hofmann, A. F., and Poley, J. R. (1972). Role of bile acid malabsorption in pathogenesis of diarrhea and steatorrhea in patients with ileal resection. Gastroenterology, 62, 918-934.

Mekhjian, H. S., and Phillips, S. F. (1970). Perfusion of the canine colon with unconjugated bile acids. Gastroenterology, 59, 120-129.

Rask-Madsen, J. (1973). Simultaneous measurement of electrical polarization and electrolyte transport by the entire normal and inflamed human colon during in vivo perfusion. Scandinavian Journal of Gastroenterology, 8, 327-336.

Rask-Madsen, J. (1974). Transepithelial ionic transport and electrical polarization in the large bowel. Scandinavian Journal of Gastroenterology, 9, 223-229. 